Relations industrielles

Industrial Relations

\title{
Danny MILLER, Peter H. FRIESEN : Organizations: A Quantum \\ View. Englewood Cliffs, Prentice-Hall, 1984, 320 pp., ISBN \\ 0-13-641985-2
}

\section{Michel Paquin}

Volume 39, numéro 3, 1984

URI : https://id.erudit.org/iderudit/050068ar

DOI : https://doi.org/10.7202/050068ar

Aller au sommaire du numéro

Éditeur(s)

Département des relations industrielles de l'Université Laval

ISSN

0034-379X (imprimé)

1703-8138 (numérique)

Découvrir la revue

Citer ce compte rendu

Paquin, M. (1984). Compte rendu de [Danny MILLER, Peter H. FRIESEN :

Organizations: A Quantum View. Englewood Cliffs, Prentice-Hall, 1984, 320 pp.,

ISBN 0-13-641985-2]. Relations industrielles / Industrial Relations, 39(3),

636-637. https://doi.org/10.7202/050068ar

Tous droits réservés (C) Département des relations industrielles de l'Université Laval, 1984
Ce document est protégé par la loi sur le droit d'auteur. L’utilisation des services d'Érudit (y compris la reproduction) est assujettie à sa politique d'utilisation que vous pouvez consulter en ligne.

https://apropos.erudit.org/fr/usagers/politique-dutilisation/ 
Workers and the New Depression, by Robert Taylor, London, Macmillan Press, 1981, pp. $\mathrm{X}+212$, ISBN 0333334116

With the heavy unemployment in the U.K. becoming a permanent phenomenon there is a justified concern how much blue collar workers suffer and where the situation leads in the long run. The author blames the lack of an adequate manpower planning, bad industrial training, excessive job restrictions and controls, as well as inability of socialists actually to represent attitudes of mind among manual workers. "Labour has proved more accomodating to the conflicting external pressures that hamper progress on the one hand, and yet more dogmatic about such nonsenses as state ownership of industry (all power to the bureaucrats) on the other» ( $p$. VIII).

With the unemployment becoming part of daily life and the lobby of protest against this fact failing to realize, there is a danger the situation to be a permanent feature. "The unemployed in Britain do not constitute a homogeneous, closely-knit group of people, capable of either organizing themselves or being mobilized to protest against this condition (...). The main victims of the slump are male manual workers without any skill or qualifications» (pp. 10, 11). In the wage jungle dictated by decentralized multi-plant bargaining, the stronger are winners and the weaker are losers. There is no hope that the better-off will make sacrifices that would benefit the weaker categories of workers. In addition, in productivity the U.K. remains much behind other countries, much suggested by the restrictive labour practices commonly appearing in various occupations. "The burdens of history - claims the author - of our voluntarist traditions and the power of the work group, remain formidable obstacles and the present slump looks unlikely to change these fundamental attitudes of mind» (p. 98).

There is no state intervention to assist these workers who can not help themselves by gaining an adequate retraining. The inequalities at work remain persistent and there is much indifference in this respect. In the unequal and divided British society strikes in reality are not an effective challenge. They «mirror a self-regarding sectionalism, with narrow aims, offering no direct challenge to the existing economic and social system» (p. 169).

The realistic perspective taken by the author helps to understand the sources of unemployment and its consequences for those people who do not have any leverage to bargain collectively for their wellbeing. There are several simplifications and misunderstandings about the fate of British unemployed which the author dismisses quite effectively. His book is a very reliable insight dictated not by specific vested interests or a doctrinaire approach but by the genuine concern for those who are neglected.

Alexander J. MATEJKO

University of Alberta

Organizations: A quantum View, by Danny Miller et Peter H. Friesen (avec la collaboration d'Henry Mintzberg), Englewood Cliffs, N.J., Prentice-Hall, 1984, 320 pp., ISBN 0-13-641985-2

La recherche traditionnelle dans le domaine des organisations a donné lieu à de nombreux débats dans la littérature. Les bureaucraties sont-elles centralisées? La proportion de personnel administratif augmente-telle avec la taille de l'organisation? Est-ce la taille ou le système technique qui est le facteur déterminant de la structure? Les résultats de ces recherches sont souvent contradictoires et le débat semble sans fin.

Pour Miller et Friesen, ces résultats décevants sont attribuables à la manière avec laquelle ces questions sont abordées. La thèse de ces auteurs est à l'effet que les méthodes traditionnellement favorisées dans l'étude des organisations encouragent l'analyse en l'absence de synthèse. Aussi, ils se font les avocats d'une approche qui favorise la synthèse en développant ou isolant des composants qui prennent la forme d'archétypes ou de configurations. Celles-ci peuvent être définies 
comme des grappes d'attributs ou de relations qui ont une forte cohésion interne, de telle sorte que la présence d'un attribut suggère, de façon fiable, la présence des autres. U1timement, le but poursuivi est de générer des typologies ou des taxonomies, c'est-à-dire des ensembles de différentes configurations qui collectivement recouvrent une fraction importante de la population des organisations considérées.

L'ouvrage de Miller et Friesen est construit autour de trois thèmes interreliés. Premièrement, les données sur les organisations peuvent révéler des structures qui ont été largement ignorées par les chercheurs. De façon spécifique, les variables de structure, d'environnement et de stratégie semblent se regrouper en configurations et un petit nombre de configurations peut être utilisé pour caractériser une fraction importante des organisations. Ceci donne aux configurations une capacité prédictive considérable. Dans la première partie de l'ouvrage, les auteurs se font les avocats de cette approche configurative et abordent la question de la méthodologie à utiliser pour découvrir les configurations. Dans la seconde partie, ils nous présentent des typologies et des taxonomies des organisations dont une typologie de la structure de l'organisation par Henry Mintzberg (les cinq configurations), une taxonomie de la décision stratégique et une taxonomie des transitions organisationnelles.

Le second thème de l'ouvrage est à l'effet que différentes configurations vont montrer plutôt des relations différentes entre leurs variables. Ainsi, il est important de distinguer entre les différentes configurations avant de faire toute prédiction entre des variables de stratégie, de structure et d'environnement. Plusieurs conflits rencontrés dans la littérature peuvent être résolus si on contrôle les données pour tenir compte du type de configuration. Ce thème est abordé dans la troisième partie de l'ouvrage et les auteurs font la démonstration de leur énoncé dans le cadre de deux études: l'une consacrée à l'étude de l'innovation dans le cas de configurations conservatrices ou entrepreneuriales; l'autre, consacrée aux déterminants de l'entrepreneurship pour trois configurations de structure.

Le thème final de l'ouvrage a trait au changement organisationnel. Il semble que l'histoire des organisations se caractérise par deux types de périodes, les plus courantes étant celles où une configuration particulière est maintenue. Ces périodes sont ponctuées par de brefs intervalles de changements importants et concertés conduisant l'organisation vers une nouvelle configuration. Cette façon d'évoluer dans le temps des organisations supporte la notion de configuration. Dans la quatrième partie de l'ouvrage, les auteurs présentent les résultats de recherches sur le changement qui soutiennent leur thèse.

L'ouvrage de Miller et Friesen est en bonne partie constitué de recherches de ces auteurs dont les résultats ont déjà été publiés. Ceci n'en fait pas pour autant un livre de peu d'intérêt. Les auteurs ont réussi à construire un ouvrage dont le contenu est bien intégré et leur thèse est des plus convaincante. Nous croyons que ce livre est important pour la recherche sur les organisations par l'influence qu'il pourrait avoir sur les méthodes utilisées par d'autres chercheurs.

\section{Michel PAQUIN}

École nationale d'administration publique

Il ruolo del fattore 'uomo' nello scenario economico-produttivo degli anni '80: i quadri, par Giuseppe De Rita, Umberto Silvestri, Michele Tedeschi, Dimitri Weiss et Shoshana Zuboff, Roma, STET, 1983, 84 p. (Introduction et conclusion de Michele Principe.)

Ce volume grand format $(30 \times 21)$, édité et diffusé (hors commerce) par les organisateurs, rassemble les Actes d'une «Rencontredébat» mise sur pied par la Direction des Problèmes syndicaux et du Travail du groupe STET, composé d'un ensemble de sociétés opérant dans les techniques de pointe (électronique, télécommunications, informatique, 